\title{
Rare Case of Intracardiac Renal Cell Carcinoma Metastasis with Response to Nivolumab: Case Report and Literature Review
}

\author{
Jawaher Ansari ${ }^{a} \quad$ Sumaya Alhelali ${ }^{b}$ Zakariya Albinmousa ${ }^{b}$ \\ Ashraf Farrag $^{c} \quad$ Arwa M. Ali $^{d}$ Mai Abdelgelil ${ }^{c} \quad$ Abdulaziz Alhamad $^{\mathrm{a}}$ \\ Ghormallah Alzahrani $^{b}$ Asif Ansarie John Glaholm ${ }^{f}$ \\ aDepartment of Oncology, Prince Sultan Military Medical City, Riyadh, Saudi Arabia; \\ bPrince Sultan Cardiac Centre, Riyadh, Saudi Arabia; ' Clinical Oncology Department, \\ Assiut University, Asyut, Egypt; ${ }^{d}$ Medical Oncology Department, South Egypt Cancer

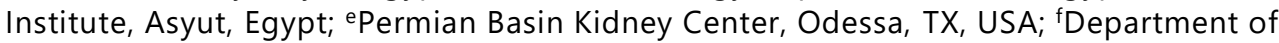 \\ Oncology, Royal Marsden Hospital, London, UK
}

\section{Keywords}

Nivolumab · Cardiac · Immunotherapy $\cdot$ Renal cell carcinoma $\cdot$ Intracardiac metastases

\begin{abstract}
Intracardiac metastases in the absence of inferior vena cava involvement is a rare occurrence in patients with metastatic renal cell carcinoma ( $\mathrm{mRCC}$ ). There is limited evidence regarding the efficacy and safety of standard treatment modalities for mRCC patients with intracardiac metastases. Presence of intracardiac metastases is known to indicate poor prognosis and may potentially increase risk of treatment-related complications. Recent advances in RCC management have integrated nivolumab, a programmed death-1 (PD-1) receptor inhibitor, as a preferred treatment option in the second-line setting after failure of prior anti-angiogenic therapy; or in combination with ipilimumab, an anti-Cytotoxic T-lymphocyte antigen-4 antibody as first-
\end{abstract}




\section{Case Reports in Oncology}

Case Rep Oncol 2018;11:861-870

DOI: $10.1159 / 000495459$

(c) 2018 The Author(s). Published by S. Karger AG, Base www.karger.com/cro

Ansari et al:: Rare Case of Intracardiac Renal Cell Carcinoma Metastasis with Response to Nivolumab: Case Report and Literature Review

line therapy for intermediate to poor risk patients with mRCC. The efficacy and toxicity of nivolumab in patients with $\mathrm{mRCC}$ and intracardiac metastases has never been reported previously. We herein present the first reported case of $\mathrm{mRCC}$ with intracardiac metastasis and a resultant excellent response to nivolumab treatment and discuss the imaging techniques and treatment options for this rare presentation.

(C) 2018 The Author(s)

Published by S. Karger AG, Basel

\section{Introduction}

Renal cell carcinoma (RCC) is the 13th most common cancer in the world, with 338,000 new cases diagnosed in 2012 [1]. Annually, there are approximately 65,000 new cases and almost 15,000 deaths from RCC in the United States [2]. Approximately $25 \%$ of RCC patients present with either locally advanced (stage III) or metastatic (stage IV) disease [3]. Historically, molecular targeted therapies have been the preferred treatment option for patients with metastatic RCC (mRCC), however, in the past 2-3 years, immunotherapy with checkpoint inhibitors has evolved as the preferred treatment option, signalling a change in the treatment paradigm. Nivolumab is a human immunoglobulin G4 monoclonal antibody, which binds to the programmed death-1 (PD-1) receptor and blocks its interaction with its ligands PD-L1 and PD-L2, potentiating T-cell responses and leading to anti-tumor activity. The United States Food and Drug Administration (FDA) initially approved nivolumab in November 2015 for the treatment of patients with advanced RCC that have received prior anti-angiogenic therapy. The approval was based on the results of the checkmate 025 trial, which demonstrated an improvement in median overall survival (25.0 vs. 19.6 months) for patients receiving nivolumab when compared to everolimus (Hazard ratio [HR] 0.73 [98.5\% CI, 0.57-0.93; $p=$ 0.002]) [4]. More recently in April 2018, the FDA approved the combination of nivolumab and ipilimumab, an anti-Cytotoxic T-lymphocyte antigen-4 (CTLA-4) antibody, for the treatment of intermediate or poor risk, previously untreated advanced RCC. The approval was based on the results of CheckMate 214 trial, which demonstrated statistically significant improvement in overall survival (HR 0.63, 95\% CI: 0.44, 0.89; $p<0.0001$ ) for patients receiving the nivolumab and ipilimumab combination when compared to sunitinib [5].

Given the expanding role of checkpoint inhibitors in mRCC it is important to be aware of its use in patients with unusual metastatic sites. The nivolumab Summary of Product Characteristics does not contain information regarding its use in RCC patients with intracardiac metastases. A published literature search in EMBASE and PubMed on 16 July 2018 using the search terms, "nivolumab/renal cell carcinoma/ cardiac/metastasis" did not identify any relevant publications. A search of www.ClinicalTrials.gov for clinical trials assessing the use of nivolumab in RCC patients with intracardiac metastases found zero results. For patients with mRCC, unusual sites of metastases may significantly influence patient treatment and prognosis. In the absence of inferior vena cava (IVC) involvement, cardiac metastases are exceptionally rare in mRCC patients with a limited number of cases reported in the literature [6-11]. We herein report the first case of a patient with mRCC and intracardiac metastasis with response to nivolumab. 


\section{Case Reports in Oncology}

Case Rep Oncol 2018;11:861-870

DOI: $10.1159 / 000495459$

(C) 2018 The Author(s). Published by S. Karger AG, Basel www.karger.com/cro

Ansari et al:: Rare Case of Intracardiac Renal Cell Carcinoma Metastasis with Response to Nivolumab: Case Report and Literature Review

\section{Case History}

A 55-year-old male presented with a 3-month history of right-sided abdominal pain. Magnetic resonance imaging (MRI) scan of the abdomen revealed a large primary tumor arising from the right kidney measuring $9.7 \times 9.2 \times 8 \mathrm{~cm}$ with invasion of the right hepatic lobe. Subsequently, a computed tomography (CT) scan of chest revealed multiple, small, bilateral pulmonary metastases. The patient's past medical history included type 2 diabetes mellitus, which was well-controlled on oral hypoglycemics. His Eastern Cooperative Oncology Group (ECOG) performance status was 0 . He underwent right-sided open cytoreductive nephrectomy along with partial resection of segment 6 of the liver. Histopathology revealed a $10-\mathrm{cm}$ clear cell RCC, Fuhrman nuclear grade 4, with evidence of sarcomatoid differentiation. There was evidence of lymphovascular invasion along with direct invasion of the liver capsule (pT4 N0 M1). The vascular, ureteric and liver parenchymal margins were negative for malignancy. Unfortunately, a follow-up CT scan performed 6-weeks later showed evidence of disease progression with an increase in the size and number of lung metastases. As per the International Metastatic RCC Database Consortium prognostic model [12], he was classed as intermediaterisk based on the presence of two adverse factors: time from diagnosis to treatment $<1$ year and serum calcium $>$ upper limit of normal (corrected calcium $=2.51 \mathrm{mmol} / \mathrm{L}$; normal range $2.03-2.44 \mathrm{mmol} / \mathrm{L})$.

He was commenced on sunitinib 50 mg once daily 4 weeks on/ 2 weeks off schedule, repeated every 6 weeks. Unfortunately, after 2 cycles of sunitinib, he developed clinical and radiological disease progression. He developed abdominal pain (score 10/10) localised to the right upper quadrant and unrelieved with opiate analgesics. His CT scan 12 weeks following initiation of sunitinib showed an increase in the size of bilateral lung metastases, development of a new right nephrectomy bed recurrence measuring $8 \times 5 \mathrm{~cm}$, along with multiple new liver metastases (Fig. 1a, 2a). In addition, a $3.4 \mathrm{~cm}$ heterogeneous filling defect was noted in the right ventricle suggestive of metastasis (Fig. 3a). Transthoracic echocardiogram showed a large echogenic mass extending from the right ventricle inflow to the right ventricular outflow tract measuring $5.6 \times 3.0 \mathrm{~cm}$ with mobile components (Fig. $4 \mathrm{a}$ ). Another large echogenic mass was seen in the right ventricular apex measuring $4.0 \times 2.2 \mathrm{~cm}$. The left and right ventricles were normal in size and systolic function with an ejection fraction of 50\%. There was no abnormality in the inferior vena cava and the pulmonary valve was not involved. The location of the intracardiac lesions in the right ventricular outflow tract and right ventricular apex were highly suggestive of metastases rather than thrombus. Cardiac MRI (CMR) performed at 1.5 Tesla, revealed a large single lesion in the right ventricle invading $47 \mathrm{~mm}$ of the inter ventricular septum and extending in to the right ventricular outflow tract with no IVC involvement highly suggestive of intracardiac metastases (Fig. 5a). Further tissue characterization of the right ventricular lesion on CMR showed the following characteristics which were diagnostic for intracardiac metastasis: (a) isointense in cine images and low intensity in pre-contrast T1weighted images, (b) High T2 signal intensity with no contrast uptake in the first pass perfusion study, and (c) heterogeneous enhancement post-contrast administration.

Differential diagnosis for the ventricular lesion included thrombus, primary or secondary tumor deposits. Biopsy of the ventricular lesion was not attempted due to the significant risk of bleeding and associated complications. Given the pattern and timing of metastatic spread during sunitinib treatment and the radiological characteristics of the intracardiac lesion, we 


\section{Case Reports in Oncology}

Case Rep Oncol 2018;11:861-870

DOI: $10.1159 / 000495459$

(C) 2018 The Author(s). Published by S. Karger AG, Basel www.karger.com/cro

Ansari et al: Rare Case of Intracardiac Renal Cell Carcinoma Metastasis with Response to Nivolumab: Case Report and Literature Review

concluded that the findings were in keeping with intracardiac metastasis. Based on the results of the Checkmate 025 trial, our preferred second-line treatment option was to consider immunotherapy with nivolumab. However, a literature review did not reveal any studies or case reports to confirm the safety or efficacy of nivolumab in patients with metastatic RCC and intracardiac metastasis.

The patient's baseline electrocardiogram showed normal sinus rhythm with no abnormalities. His blood pressure was normal at $124 / 70 \mathrm{~mm}$ of $\mathrm{Hg}$. He was commenced on nivolumab $240 \mathrm{mg}$ intravenously every 2 weeks. Two weeks after commencement of nivolumab, the patient noted a significant improvement in his pain control (pain score 2/10) along with $70 \%$ reduction in his opiate requirement. Eight weeks later, his follow-up CT scan demonstrated a partial response to nivolumab with a significant reduction in the size of liver metastases (Fig. 1b), lung metastases and nephrectomy bed recurrence (Fig. 2b). In addition, the CT scan also showed a significant reduction in the size of the intracardiac metastasis (Fig. $3 b)$. Follow-up transthoracic echocardiogram showed a $60 \%$ reduction in the size of the intracardiac metastasis (Fig. 4b). CMR at week 18 of nivolumab showed $44 \%$ reduction in the size of the intracardiac metastasis from $47 \times 25$ to $21 \times 7.9 \mathrm{~mm}$ with development of central necrosis, indicating an excellent response to nivolumab (Fig. 5b). Nivolumab treatment was well-tolerated with no treatment-related adverse events. Twelve months following initiation of nivolumab, his follow-up CT scan has shown evidence of continued response (Fig. 3c). The patient's performance status has improved to 0 and he is currently continuing treatment with nivolumab.

\section{Discussion}

Immunotherapy with nivolumab has become an integral treatment option in the management pathway for patients with mRCC. Currently, there is limited evidence regarding the efficacy and safety of checkpoint inhibitors for patients with unusual metastatic sites such as intracardiac metastases. Cardiac involvement in RCC commonly arises from direct tumor thrombus extension into the IVC in approximately $5-10 \%$ of patients. However, cardiac involvement in the absence of IVC involvement is extremely rare and poses a unique therapeutic challenge. In a pooled retrospective analysis of mRCC patients treated in 4 clinical trials $(n=$ 1,765), the incidence of intracardiac metastases without IVC involvement was seen in $<1 \%$ ( $n$ $=10$ ) [13]. In this study, molecular targeted therapy resulted in partial response in $10 \%$ and stable disease in $60 \%$ of patients with a median progression-free survival of 6.9 months, demonstrating poor outcomes for patients with intracardiac metastases. None of the patients in this study were treated with checkpoint inhibitors.

Cases of mRCC with intracardiac metastases without IVC involvement and documented systemic treatment outcomes have been summarized in Table 1.

Expert recommendations for patients with mRCC state that for patients with an acute cardiac event within the previous 6 months, New York Heart Association grade III heart failure, or those with uncontrolled high blood pressure should not be treated with tyrosine kinase inhibitors [21]. There are no specific guidelines or recommendations for nivolumab in patients with pre-existing cardiovascular co-morbidities. Although, fatal myocarditis has been reported after a single dose of the combination of nivolumab and ipilimumab [22]. However, 


\section{Case Reports in Oncology}

Case Rep Oncol 2018;11:861-870

DOI: $10.1159 / 000495459$

(C) 2018 The Author(s). Published by S. Karger AG, Basel www.karger.com/cro

Ansari et al.: Rare Case of Intracardiac Renal Cell Carcinoma Metastasis with Response to Nivolumab: Case Report and Literature Review

in pharmacovigilance studies, the incidence of myocarditis was higher in patients treated with the combination of nivolumab plus ipilimumab as compared with nivolumab alone $0.27 \mathrm{vs}$. $0.06 \%$ ). Although, the incidence of nivolumab-induced cardiac toxicity is rare but nevertheless could be relevant in patients with pre-existing cardiac issues such as our patient. Clinical trials that included patients with intracardiac metastases did not report any increase in cardiovascular toxicity for patients treated with tyrosine kinase inhibitors, however, similar data for checkpoint inhibitors is lacking [13].

Patients with intracardiac metastases may present with haemodynamic consequences due to ventricular outflow obstruction, embolism and electrical or mechanical cardiac dysfunction. As symptoms may be largely non-specific, a high index of suspicion is needed to guide relevant investigations. For patients with suspected intracardiac metastases, transthoracic echocardiography is the initial recommended imaging modality to detect the location, size, and mobility of the cardiac lesions. However, echocardiography has its limitations in assessing patients with poor acoustic windows and for assessment of extra-cardiac structures [23]. Echocardiographic findings in favour of intracardiac metastases include: presence of multiple lesions, location in right ventricle, and well-circumscribed margins [24]. Although tissue histology remains the gold standard to diagnose cardiac metastases, it is not always necessary or technically feasible and could potentially be associated with significant morbidity and mortality given that RCC metastases are typically very vascular [25]. Modern imaging techniques such CMR and 18 F-fluorodeoxyglucose positron emission tomography CT (PETCT) can provide additional non-invasive characterization with a high degree of specificity for suspected intracardiac metastases. CT scans have good spatial resolution and are useful to rule out coronary artery involvement [26]. PET-CT can further differentiate between benign and malignant intracardiac lesions based on FDG uptake [27]. CMR signal intensity can provide essential tissue characterisation that is needed to differentiate between intracardiac metastases and other possible differential diagnoses such as tumour thrombus, myxoma, sarcoma or other primary tumours. CMR features that support the diagnosis of intracardiac metastases include- hypointensity on T1-weighted images, hyperintensity on T2-weighted images and heterogeneous enhancement with contrast [28].

Nivolumab has shown promising results in the first- and second-line setting for patients with mRCC. Historically, patients with cardiac metastases are thought to have poor prognosis but this may no longer be the case in the immunotherapy era. Our case report adds further to literature by highlighting the usage of nivolumab in this rare subpopulation of patients with mRCC and intracardiac metastases without IVC involvement.

\section{Conclusion}

Patients with mRCC and intracardiac metastases without IVC involvement are considered extremely rare. Outcomes for this subpopulation demonstrate poor outcomes in the era of targeted therapy. However, this may no longer be the case with the expanding role of checkpoint inhibitors in mRCC management. This case highlights the rare presentation of mRCC with intracardiac metastasis without IVC involvement and demonstrates an excellent response to treatment with nivolumab. Our case provides valuable information on the 
tolerability, long-term safety and efficacy of nivolumab for patients with mRCC with intracardiac metastases.

\section{Statement of Ethics}

An informed consent was obtained from the patient for the publication of his information and imaging. The authors have no ethical conflicts to report.

\section{Disclosure Statement}

Dr. Jawaher Ansari and Dr. Abdulaziz Alhamad have received lecture fees from BristolMyers Squibb. Dr. Sumaya Alhelali, Dr. Zakariya Albinmousa, Dr. Ashraf Farrag, Dr. Arwa Ali, Dr. Mai Abdelgelil, Dr. Ghormallah Alzahrani, Dr. Asif Ansari, and Dr. John Glaholm have no conflict of interest to declare.

\section{References}

1 Ferlay J, Soerjomataram I, Dikshit R, Eser S, Mathers C, Rebelo M, et al. Cancer incidence and mortality worldwide: sources, methods and major patterns in GLOBOCAN 2012. Int J Cancer. 2015 Mar;136(5):E35986.

2 Siegel RL, Miller KD, Jemal A. Cancer statistics, 2018. CA Cancer J Clin. 2018 Jan;68(1):7-30.

3 Cohen HT, McGovern FJ. Renal-cell carcinoma. N Engl J Med. 2005 Dec;353(23):2477-90.

4 Motzer RJ, Escudier B, McDermott DF, George S, Hammers HJ, Srinivas S, et al.; CheckMate 025 Investigators. Nivolumab versus Everolimus in Advanced Renal-Cell Carcinoma. N Engl J Med. 2015 Nov;373(19):1803-13.

5 Motzer RJ, Tannir NM, McDermott DF, Arén Frontera O, Melichar B, Choueiri TK, et al.; CheckMate 214 Investigators. Nivolumab plus Ipilimumab versus Sunitinib in Advanced Renal-Cell Carcinoma. N Engl J Med. 2018 Apr;378(14):1277-90.

6 Briasoulis A, Siddiqui F, Siddiqui S, Mohamad T, Afonso L. Large right ventricular mass causing outflow tract obstruction secondary to metastatic renal cell carcinoma. Acta Cardiol. 2015 Feb;70(1):86-7.

7 Zustovich F, Gottardo F, De Zorzi L, Cecchetto A, Dal Bianco M, Mauro E, et al. Cardiac metastasis from renal cell carcinoma without inferior vena involvement: a review of the literature based on a case report. Two different patterns of spread? Int J Clin Oncol. 2008 Jun;13(3):271-4.

8 Atik FA, Navia JL, Krishnamurthi V, Singh G, Shiota T, Pitas G, et al. Solitary massive right ventricular metastasis of renal cell carcinoma without inferior vena cava or right atrium involvement. J Card Surg. 2006 May-Jun;21(3):304-6.

9 Sato T, Takeda A, Yamada S, Numata I, Sakamoto K. Metastatic renal cell carcinoma to right ventricle without vena caval involvement. Int J Urol. 2008 Apr;15(4):366-8.

10 Carroll JC, Quinn CC, Weitzel J, Sant GR. Metastatic renal cell carcinoma to the right cardiac ventricle without contiguous vena caval involvement. J Urol. 1994 Jan;151(1):133-4.

11 Schinzari G, Monterisi S, Signorelli D, Cona MS, Cassano A, Danza F, et al. Cardiac metastasis from renal cell carcinoma successfully treated with pazopanib: impact of TKIs' antiangiogenic activity. Tumori. 2014 NovDec;100(6):e298-300.

12 Heng DY, Xie W, Regan MM, Harshman LC, Bjarnason GA, Vaishampayan UN, et al. External validation and comparison with other models of the International Metastatic Renal-Cell Carcinoma Database Consortium prognostic model: a population-based study. Lancet Oncol. 2013 Feb;14(2):141-8.

13 Viteri Malone MA, Ares GR, De Velasco G, Brandão R, Lin X, Norton C, et al. The Clinical Presentation, Survival Outcomes, and Management of Patients With Renal Cell Carcinoma and Cardiac Metastasis Without Inferior Vena Cava Involvement: Results From a Pooled Clinical Trial Database and Systematic Review of Reported Cases. Clin Genitourin Cancer. 2018 Apr;16(2):e327-33. 
Ansari et al.: Rare Case of Intracardiac Renal Cell Carcinoma Metastasis with Response to Nivolumab: Case Report and Literature Review

14 Bazine A, Fetohi M, Tanz R, Mahfoud T, Ichou M, Errihani H. Cardiac metastases of renal cell carcinoma revealed by syncope: diagnosis and treatment. Case Rep Oncol. 2014 Aug;7(2):560-4.

15 Abdullah A, Lekkala M, Wolfe Z, Raghu C, Khan SU, Krishnan M, et al. Isolated Left Ventricular Metastasis from Renal Cell Carcinoma: Diagnostic and Therapeutic Dilemma. Case Rep Oncol. 2018 Jun;11(2):365-71.

16 Szmit S, Zagrodzka M, Kurzyna M, Opolski G, Szczylik C. Sunitinib malate, a receptor tyrosine kinase inhibitor, is effective in the treatment of restrictive heart failure due to heart metastases from renal cell carcinoma. Cardiology. 2009;114(1):67-71.

17 Czarnecka AM, Sobczuk P, Lian F, Szczylik C. Renal cell carcinoma with intramyocardial metastases. BMC Urol. 2014 Sep;14(1):73.

18 Zhang B, Malouf J, Young P, Kohli M, Dronca R. Cardiac Metastasis in Renal Cell Carcinoma without Vena Cava or Atrial Involvement: an Unusual Presentation of Metastatic Disease. Rare Tumors. 2013 Jul;5(3):e29.

19 Tatenuma T, Yao M, Sakata R, Sano F, Makiyama K, Nakaigawa N, et al. [A case of myocardiac metastasis of clear cell renal carcinoma successfully treated with sunitinib]. Hinyokika Kiyo. 2013 Feb;59(2):97-101.

20 Satpathy R, Lynch J, Mohiuddin SM. Ventricular metastasis without atrial or caval involvement: a rare presentation. Echocardiography. 2008 May;25(5):521-5.

21 Puente J, García Del Muro X, Pinto Á, Láinez N, Esteban E, Arranz JÁ, et al. Expert Recommendations for FirstLine Management of Metastatic Renal Cell Carcinoma in Special Subpopulations. Target Oncol. 2016 Apr;11(2):129-41.

22 Johnson DB, Balko JM, Compton ML, Chalkias S, Gorham J, Xu Y, et al. Fulminant Myocarditis with Combination Immune Checkpoint Blockade. N Engl J Med. 2016 Nov;375(18):1749-55.

23 Goldberg AD, Blankstein R, Padera RF. Tumors metastatic to the heart. Circulation. 2013 Oct;128(16):17904.

24 Basso C, Rizzo S, Valente M, Thiene G. Cardiac masses and tumours. Heart. 2016 Aug;102(15):1230-45.

25 Curci N, Caoili EM. The Current Role of Biopsy in the Diagnosis of Renal Tumors. Semin Ultrasound CT MR. 2017 Feb;38(1):72-7.

26 Chiles C, Woodard PK, Gutierrez FR, Link KM. Metastatic involvement of the heart and pericardium: CT and MR imaging. Radiographics. 2001 Mar-Apr;21(2):439-49.

27 Rahbar K, Seifarth H, Schäfers M, Stegger L, Hoffmeier A, Spieker T, et al. Differentiation of malignant and benign cardiac tumors using 18F-FDG PET/CT. J Nucl Med. 2012 Jun;53(6):856-63.

28 Fussen S, De Boeck BW, Zellweger MJ, Bremerich J, Goetschalckx K, Zuber M, et al. Cardiovascular magnetic resonance imaging for diagnosis and clinical management of suspected cardiac masses and tumours. Eur Heart J. 2011 Jun;32(12):1551-60.
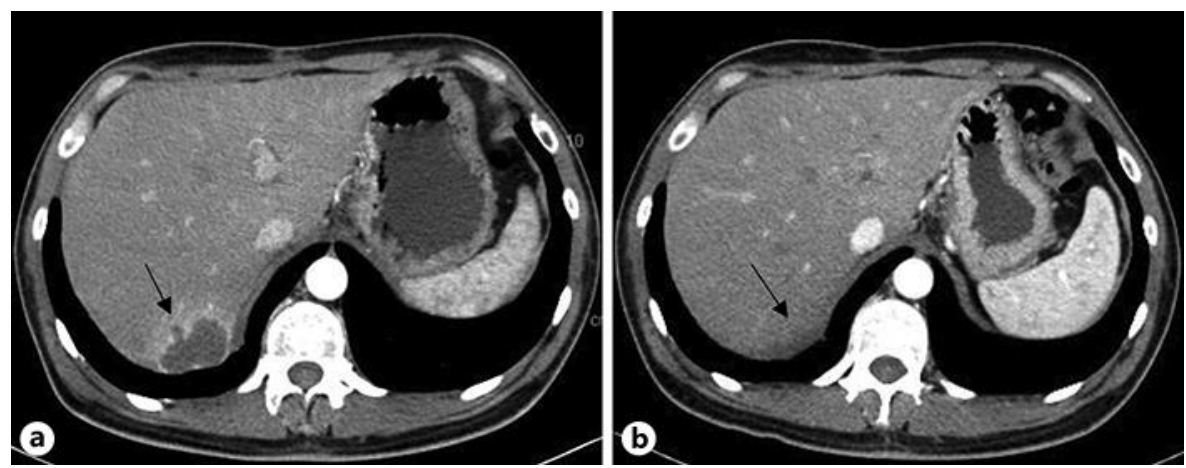

Fig. 1. a, b: Axial contrast enhanced computed tomography scan of the abdomen (a) at baseline demonstrating a $2.8 \times 4.6 \mathrm{~cm}$ metastasis in segment 7 of the liver (b) following 8-weeks of nivolumab treatment showing a partial response of the liver metastasis. 


\section{Case Reports in Oncology}
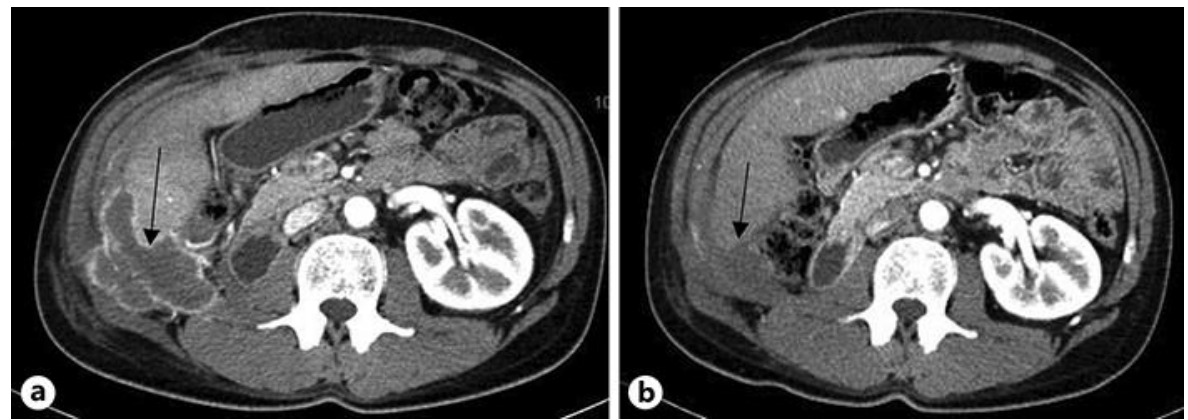

Fig. 2. a, b: Axial contrast enhanced computed tomography scan of the abdomen (a) at baseline demonstrating an $8 \times 5 \mathrm{~cm}$ local recurrence over the right renal bed invading the liver, abdominal wall and iliopsoas muscles (b) following 8-weeks of nivolumab treatment showing a partial response with a significant reduction in the size of the right renal bed recurrence.
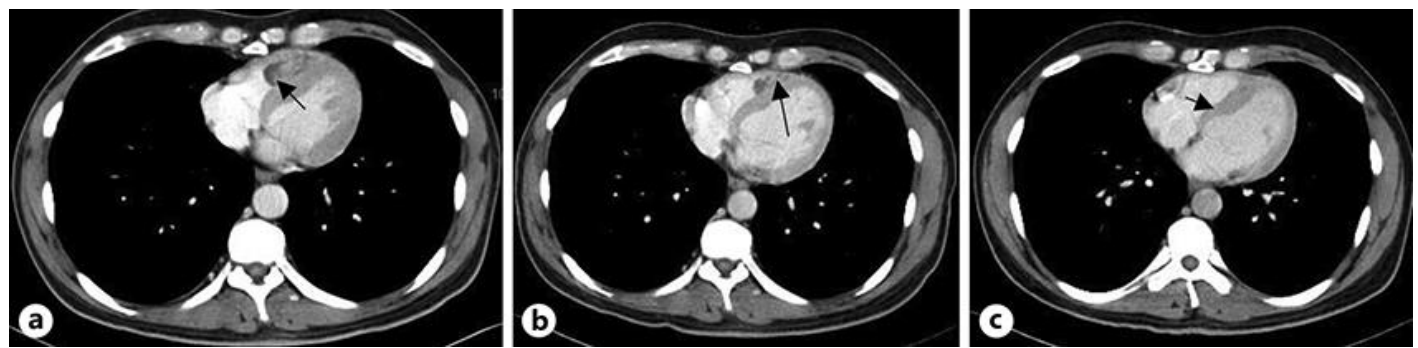

Fig. 3. a-c: Axial contrast enhanced computed tomography scan of the chest (a) at baseline demonstrating a $3.4 \mathrm{~cm}$ heterogeneous filling defect in the right ventricle suggestive of intracardiac metastasis (b) following 8-weeks of nivolumab treatment showing mild reduction in the size of the intracardiac metastases (c) following 12-months of nivolumab treatment showing a $70 \%$ reduction in the size of the intracardiac metastasis now measuring $0.7 \times 0.7 \mathrm{~cm}$. 


\section{Case Reports in Oncology}
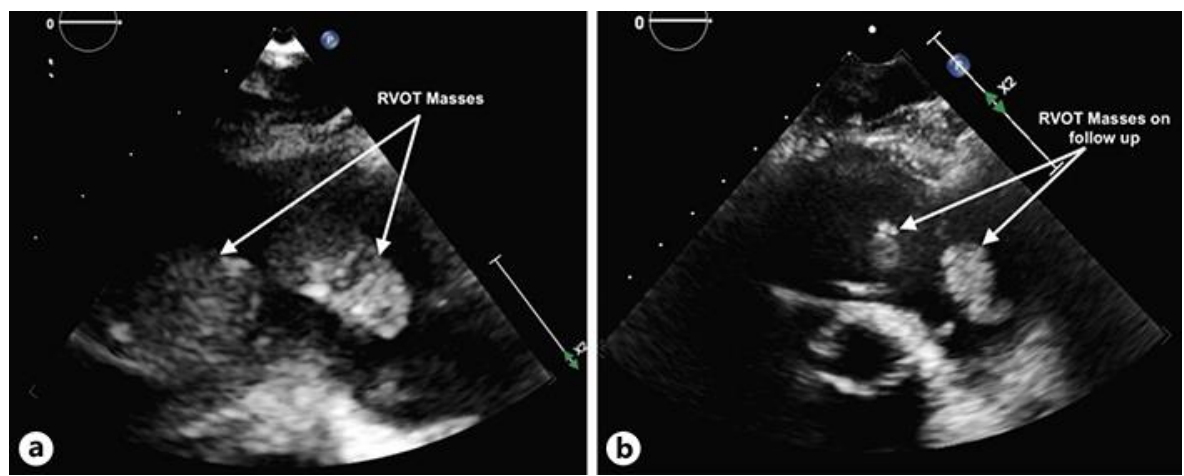

Fig. 4. a, b: Parasternal short axis view transthoracic echocardiogram of the right ventricular outlet tract (RVOT) (a) at baseline showing two large intracardiac metastases (b) following 8-weeks of nivolumab treatment showing $60 \%$ reduction in the size of the intracardiac metastases.
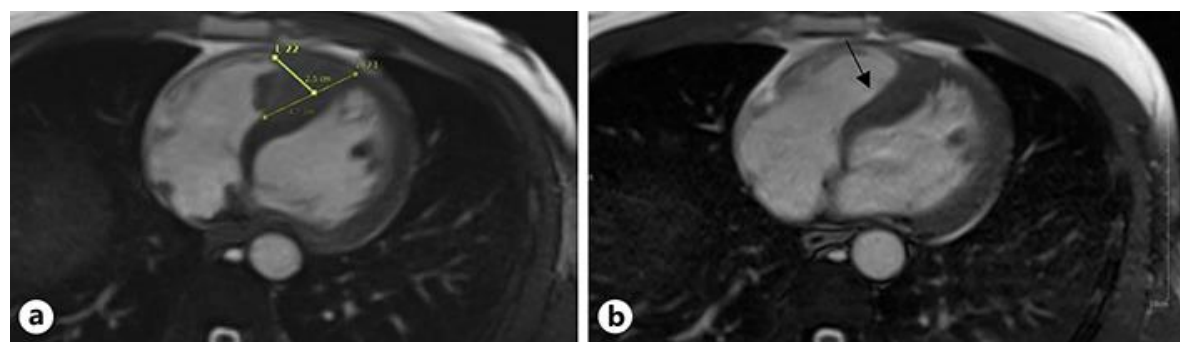

Fig. 5. a, b: Cardiac magnetic resonance imaging axial cine images showing (a) A large metastatic deposit in the right ventricle with multiple components invading $47 \mathrm{~mm}$ of the right-sided interventricular septum (b) following 18-weeks of nivolumab treatment showing $44 \%$ reduction in the size of the intracardiac metastasis. 
Table 1. Summary of reported cases highlighting systemic treatment outcomes for patients with mRCC with intracardiac metastases without IVC involvement [14-20]

\begin{tabular}{|c|c|c|c|c|}
\hline Reference & Age & Sex & Location & Systemic treatment and response \\
\hline Bazine et al. [14] & 60 & M & Right ventricle & $\begin{array}{l}\text { 1st line: Sunitinib-PD } \\
\text { 2nd line: Everolimus-PD }\end{array}$ \\
\hline Abdullah et al. [15] & 74 & M & Left ventricle & Pazopanib-PR $\times 5$ months \\
\hline Szmit et al. [16] & 61 & M & Left atrium & Sunitinib-PR \\
\hline Czarnecka et al. [17] & 50 & M & Left ventricle & Pazopanib-SD \\
\hline Zhang et al. [18] & 70 & $\mathrm{M}$ & Left ventricle & $\begin{array}{l}\text { 1st line:Temsirolimus-SD } \times 5 \text { months } \\
\text { 2nd line: Sunitinib-SD } \times 7 \text { months }\end{array}$ \\
\hline Zhang et al. [18] & 64 & M & Right ventricle & 2) Sunitinib-SD $\times 2$ years \\
\hline Tatenuma et al. [19] & 65 & M & Right ventricle & Sunitinib $-\mathrm{PR} \times 6$ months \\
\hline Satpathy et al. [20] & 44 & M & $\begin{array}{l}\text { Left and right } \\
\text { ventricles }\end{array}$ & Interleukin-1: PR \\
\hline
\end{tabular}

PD, progressive disease; PR, partial response; SD, stable disease. 